\title{
Constitutive relations for the shear-band evolution in granular matter under large strain
}

\author{
Stefan Luding \\ Multi Scale Mechanics, TS, CTW, UTwente \\ POBox 217, 7500 AE Enschede, Netherlands \\ s.luding@utwente.nl
}

July 18, 2008

\begin{abstract}
A so-called "split-bottom ring shear cell" leads to wide shear bands under slow, quasi-static deformation. From discrete element simulations (DEM), several continuum fields like the density, velocity, deformation gradient and stress are computed and evaluated with the goal to formulate objective constitutive relations for the powder flow behavior. From a single simulation, by applying time- and (local) space-averaging, a nonlinear yield surface is obtained with a peculiar stress dependence.

The anisotropy is always smaller than the macroscopic friction coefficient. However, the lower bound of anisotropy increases with the strain rate, approaching the maximum according to a stretched exponential with a specific rate that is consistent with a shear path of about one particle diameter.
\end{abstract}

\section{Introduction}

Granular Matter consists of many independent particles with peculiar collective flow behavior. Knowing the interaction laws and inserting those into a discrete element model (DEM), one can follow the particles by integrating Newtons equations of motion (Herrmann et al., 1998; Kishino, 2001; Luding et al., 2001; Luding, 2004b; Luding, 2008b).

One goal is to derive continuum constitutive relations - as needed for industrial application. Methods and tools for a so-called micro-macro transition 
are applied (Vermeer et al., 2001; Lätzel et al., 2000; Luding, 2004a; Luding, 2005b; Luding, 2005a; Luding, 2008b) on small so-called representative volume elements (RVE). In ring-shear cells, both local space averaging (on toroidal sub-volumes at fixed radial and vertical position) as well as time-averaging in the (presumed) steady state can be applied. One obtains already from a single simulation some of the constitutive relations aimed for. Here, the micro-macro averaging is applied to a three-dimensional splitbottom shear cell as recently presented (Fenistein and van Hecke, 2003; Fenistein et al., 2004). The special property of a split-bottom ring shear cell is the fact that the shear band is initiated at the bottom slit and its velocity field is well approximated by an error-function (Fenistein et al., 2004; Luding, 2004b; Luding, 2006) with a width considerably increasing from bottom to top (free surface). In this study, the frictionless data are examined closer and the stress- and strain-tensors are studied in their eigensystems and eigen-directions. A recently proposed evolution equation for the deviatoric stress (Luding, 2008c) is examined here.

\section{The Soft Particle Molecular Dynamics Method}

The behavior of granular media can be simulated with the discrete element method (DEM) (Allen and Tildesley, 1987; Lätzel et al., 2003; Luding, 2008a). As the basic ingredient, a force-displacement relation that governs the interaction between pairs of particles is defined. Particle positions, velocities and interaction forces are then sufficient to integrate (explicitly) Newtons equations of motion and follow all particles during the evolution of the system under large strains.

Since the modeling of the internal deformations of the particles is much too complicated, we relate the normal interaction force to the overlap as $f=k \delta$, with a stiffness $k$, if $\delta>0$. In order to account for energy dissipation, the normal degrees of freedom, i.e. the relative motion of two particles in contact, is subject to a viscous, velocity dependent damping, for more details see (Luding, 1998; Luding, 2008a).

\section{Split-bottom ring shear cell}

In order to save computing time, only a quarter of the ring-shaped geometry is simulated, using quarter periodic boundary conditions in angular direction. (In top-view, a particle that leaves the quarter system downwards, enters at the same radial position from the right - with according, 
unchanged velocity in cylindrical coordinates). The walls are cylindrical, and are roughened due to some (about 3 per-cent of the total number) attached particles (Luding, 2004b; Luding, 2006; Luding, 2008b; Luding, 2008c). The outer cylinder wall with radius $R_{o}=0.110 \mathrm{~m}$, and part of the bottom $r>R_{s}=0.085 \mathrm{~m}$ are rotating around the symmetry axis with the same rotation rate, while the inner wall with radius $R_{i}=0.0147 \mathrm{~m}$, and the attached bottom-disk $r<R_{s}$ remain at rest.

First, the simulation runs for more than $50 \mathrm{~s}$ with a rotation rate $f_{o}=0.01 \mathrm{~s}^{-1}$ of the outer cylinder, with angular velocity $\Omega_{o}=2 \pi f_{o}$. For the average only larger times are taken into account, thus disregarding the transient behavior at the onset of shear. Two snapshots (top and front view) are displayed in Fig. 1.
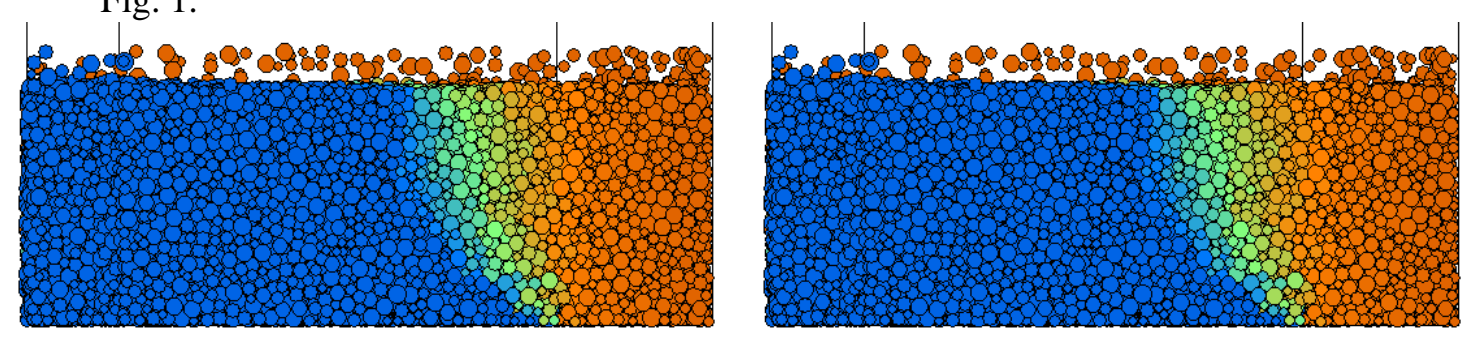

Figure 1: Snapshots from a simulation with $N=34518$ particles, without friction $\mu=0$. The colors blue, green, orange and red denote particles with displacements in tangential direction per second $r \mathrm{~d} \phi \leq 0.5 \mathrm{~mm}, r \mathrm{~d} \phi \leq 2 \mathrm{~mm}, r \mathrm{~d} \phi \leq 4 \mathrm{~mm}$, and $r \mathrm{~d} \phi>4 \mathrm{~mm}$.

Translational invariance is assumed in the tangential $\phi$-direction, and averaging is thus performed over toroidal volumina over many snapshots in time (typically 40-60), leading to fields $Q(r, z)$ as function of the radial and vertical positions. Here, averaging is performed with spacings of $\Delta r \approx$ 0.0025 and $\Delta z \approx 0.0028$ in radial and vertical direction. The choice of these spacings is arbitrary, since they do not affect the results discussed below if varied somewhat. However, much smaller spacing leads to bad statistics and stronger fluctuations while much larger spacing leads to poor resolution and thus loss of information.

The averaged data from simulations lead to density, coordination number, and the isotropic fabric, all decreasing with height and systematically lower in the shear band due to dilatancy. From a set of simulations with different filling heights (data not shown, see (Luding, 2004b)), just examined from the top (like in the original experiments), it becomes clear that the shearband moves inwards with increasing filling height and also becomes wider. From the front-view, the same information can be evidenced, see Fig. 1, as well as 
shear band shape and width inside the bulk. The shear band moves rapidly inwards deep in system - close to the slit in the bottom - while its position does not change much more further up.

From the velocity field gradient, the strain rate

$$
\dot{\gamma}=\sqrt{d_{1}^{2}+d_{2}^{2}}=\frac{1}{2} \sqrt{\left(\frac{\partial v_{\phi}}{\partial r}-\frac{v_{\phi}}{r}\right)^{2}+\left(\frac{\partial v_{\phi}}{\partial z}\right)^{2}},
$$

is obtained, as discussed in Ref. (Depken et al., 2006), see Eq. (7) therein, where the geometrical term, $v_{\phi} / r$ in Eq. (1), comes from the cylindrical coordinate system. From the eigenvalue analysis of the velocity gradient, one finds that shear planes are well described by the normal unit vector $\hat{\gamma}=(\cos \theta, 0, \sin \theta)$, with $\theta=\theta(r, z)=\arccos \left(d_{1} / \dot{\gamma}\right)$, as predicted in Ref. (Depken et al., 2006). This unit-vector, $\hat{\gamma}$, is the eigen-vector of the vanishing eigenvalue of the velocity gradient tensor, while the other two are opposite-equal, with their eigen-vectors in the plane perpendicular to $\hat{\gamma}$. From the simulation, one can determine the components of the static stress

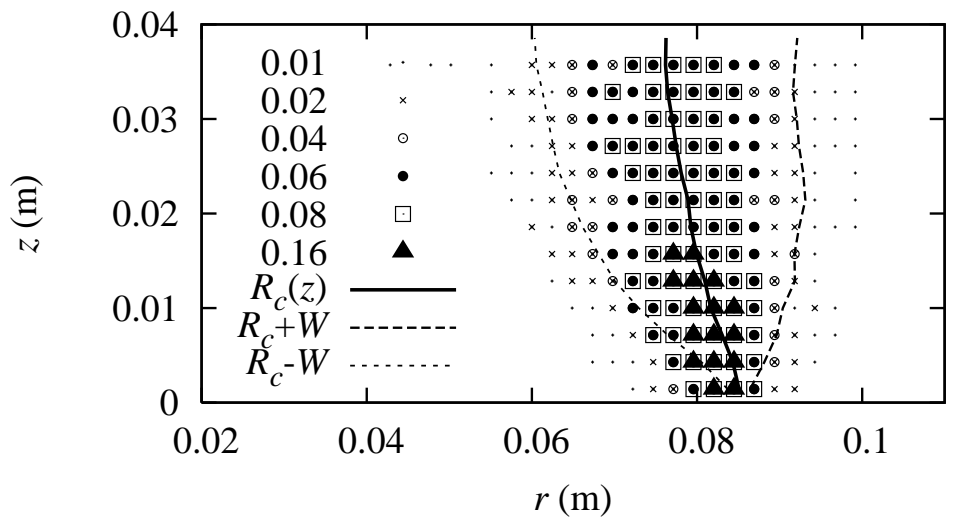

Figure 2: Graphic representation of the strain rate $\dot{\gamma}$, with the values given in the inset, plotted as function of radial and vertical positions. The solid line indicates the center $R_{c}$ and the dashed lines show plus-minus the half-width $W$, as obtained by fitting an error-function to the velocity data

tensor

$$
\sigma_{\alpha \beta}=\frac{1}{V} \sum_{c \in V} f_{\alpha} l_{\beta}
$$

with the contact normal forces $f_{\alpha}$ and branch vector $l_{\beta}$ components. The sum includes contacts in the vicinity of the averaging volume, $V$, weighted according to their vicinity. 
Since the $\sigma_{r z} \approx 0$ component is small, as compared to the other averaged non-diagonal stresses, the shear stress can be defined in analogy to the velocity gradient, as proposed in (Depken et al., 2006):

$$
|\tau|=\sqrt{\sigma_{r \phi}^{2}+\sigma_{z \phi}^{2}} .
$$

A more detailed study of the stress- and strain eigenvalues and eigensystems leads to the three eigenvalues $\sigma_{\max }, \sigma_{0}$, and $\sigma_{\min }$ corresponding to the maximum, intermediate and minimum stress, respectively, with corresponding eigen-directions $\hat{\sigma}_{\max }, \hat{\sigma}_{0}$, and $\hat{\sigma}_{\text {min. }}$. In Fig. 4 , the shear stress $|\tau|$ and the deviator stress

$$
\sigma_{D}=\sqrt{\left(\sigma_{\max }-\sigma_{\min }\right)^{2}+\left(\sigma_{\max }-\sigma_{0}\right)^{2}+\left(\sigma_{0}-\sigma_{\min }\right)^{2}} / \sqrt{6}
$$

are plotted against the pressure $p=\left(\sigma_{\max }+\sigma_{0}+\sigma_{\min }\right) / 3$. Note that the definition of $\sigma_{D}$ is equivalent to $q=\left(\sigma_{\max }-\sigma_{\min }\right) / 2$ in the case of a stress tensor with $\sigma_{0}=\left(\sigma_{\max }-\sigma_{\min }\right) / 2$.

Shear stress, $\tau$, and deviator $\sigma_{D}$ quantify the stress anisotropy and are almost identical, see Figs. 3 and 4 , only $\sigma_{D}$ appears systematically somewhat larger than $|\tau|$. The ratio $\sigma_{D} / \tau>1$ is decaying with the strain rate (data not shown here - but note the big scatter) and indicates how good the stress tensor conforms with the assumptions that lead to Eq. (3) - if the strain rate is large.

From the (almost) constant shear stress intensity and deviator stress in the shear zone, one can determine the Mohr-Coulomb-type friction angle of the equivalent macroscopic constitutive law, as $\psi \approx \arcsin \mu_{\text {macro. Interest- }}$ ingly, without friction $\psi$ is rather large, i.e., much larger than expected from a frictionless material, whereas it is rather small with a friction coefficient as used in Ref. (Luding, 2008b) (data not shown here). Similar behavior was already observed in two-dimensional bi-axial shear tests (?). There the critical state macroscopic friction coefficient is finite for small contact friction $\mu \rightarrow 0$ under a finite confining stress, and saturates at a rather small value for large contact friction $\mu>0.2$. A detailed study of different coefficients of contact friction is far from the scope of this study.

From Fig. 4, one observes that the anisotropy is stress dependent, increasing up to moderate stress levels, and then remaining more or less constant within the fluctuations.

Plotting the shear stress intensity $|\tau| / p$ and the anisotropy $\sigma_{D} / p$ against either the strain rate $\dot{\gamma}$, or against the non-dimensional strain rate

$$
I=\dot{\gamma} d_{0} \sqrt{\varrho / p},
$$



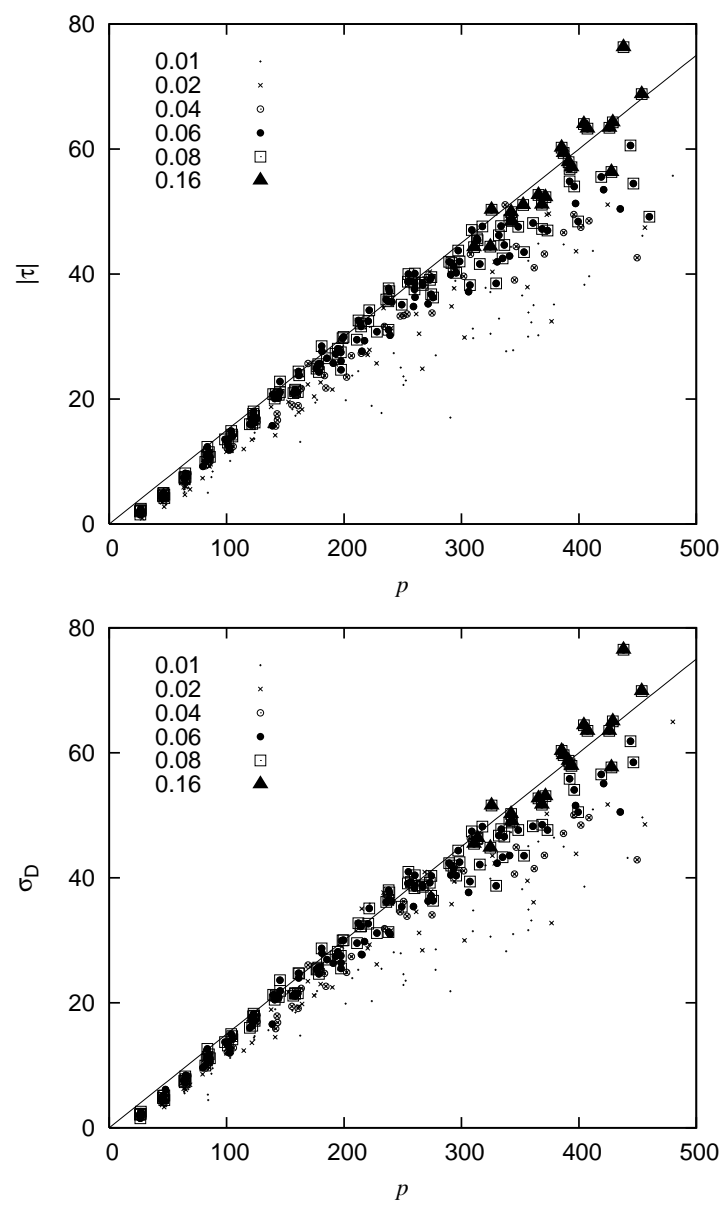

Figure 3: Shear stress $|\tau|$ and deviator stress $\sigma_{D}$, plotted against pressure $p$ for different strain rates as given in the inset. All points with large strain rate are found close to the yield surface $\mu_{\text {macro }} p$, as represented by the solid line, with constant $\mu_{\text {macro }}=0.15$. 

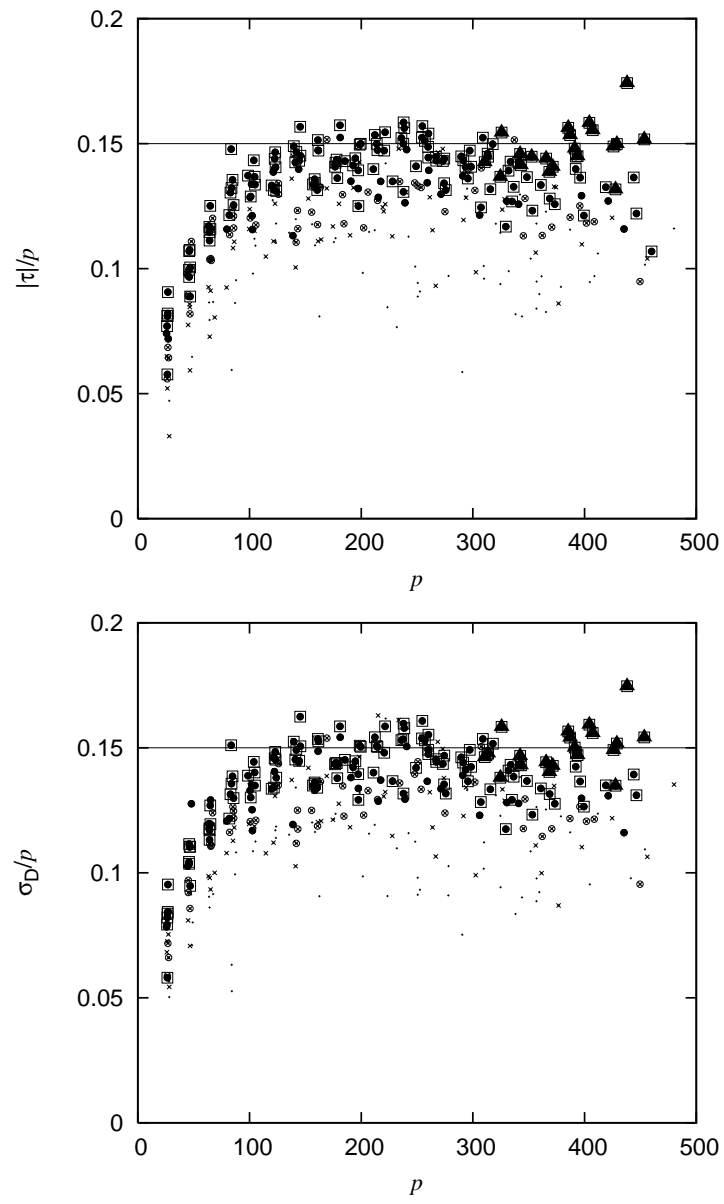

Figure 4: (Left) Shear stress ratio $|\tau| / p$ and (Right) anisotropy $\sigma_{D} / p$, plotted against pressure $p$ for different strain rates as given in the inset. 
with the mean particle diameter $d_{0}$, and the bulk density, $\varrho$, as proposed in Ref. (MiDi, 2004), neither leads to a better trend nor a better data collapse. Therefore, we define the dimensionless shear path

$$
l_{\gamma}=\Delta t \dot{\gamma},
$$

with the simulation averaging time interval $\Delta t=25.4 \mathrm{~s}$, used for averaging. The shear-path, $l_{\gamma}$, indicates about how far the shear planes have been sheared relative to each other. Note that a different definition was used in Ref. (Luding, 2008c).

When plotting $|\tau| / p$ and $s_{D}:=\sigma_{D} / p$, in Fig. 5, against the shear path, $l_{\gamma}$, the former appears (again) somewhat smaller than the latter. The question whether the very small systematic discrepancy between shear stress and deviator stress have a physical meaning or are only a consequence of statistical fluctuations can only be answered by a more careful analysis of the stress, the fabric, and the velocity gradient, which is far from the scope of this study.

Besides their scatter, the data in Fig. 5 (Right) fall between the maximum anisotropy, $\mu_{\text {macro }}=0.15$, and the lower bound $s_{D}^{\min }$ so that:

$$
\mu_{\text {macro }} \geq s_{D}>s_{D}^{\min }:=\mu_{\text {macro }}\left[1-\exp \left(-l_{\gamma}^{\alpha}\right)\right],
$$

where the exponent $\alpha \approx 0.5$ seems to be a better choice than $\alpha=1$. Note, however, that this is only an empirical fit-function without a theoretical basis (yet). The stretched exponential lower bound indicates slow relaxation processes, which require that the granular material shear planes move along each other for a certain distance, before the steady state shear regime with anisotropy $s_{D} \approx \mu_{\text {macro }}$ can be reached.

An initial (local) anisotropy, $0 \leq s_{D}^{0} \leq \mu_{\text {macro }}$, could evolve according to the evolution equation

$$
\frac{\partial s_{D}}{\partial l_{\gamma}}=\left(\mu_{\text {macro }}-s_{D}\right)
$$

which would be consistent with $\alpha=1$, as observed from two-dimensional simulations and experiments, see Ref. (Luding, 2004a) and references therein. A quantitative confirmation of the above evolution law for the stress anisotropy with shear deformation is subject to further study of both steady and transient states.

Using the functional form of the lower bound stress anisotropy from Eq. (6), for arbitrary initial $s_{D}^{0}$, leads to the anisotropy evolution with shear path:

$$
s_{D}\left(l_{\gamma}\right)=\mu_{\text {macro }}\left[1-\left(\mu_{\text {macro }}-s_{D}^{0}\right) \exp \left(-l_{\gamma}^{\alpha}\right)\right] .
$$



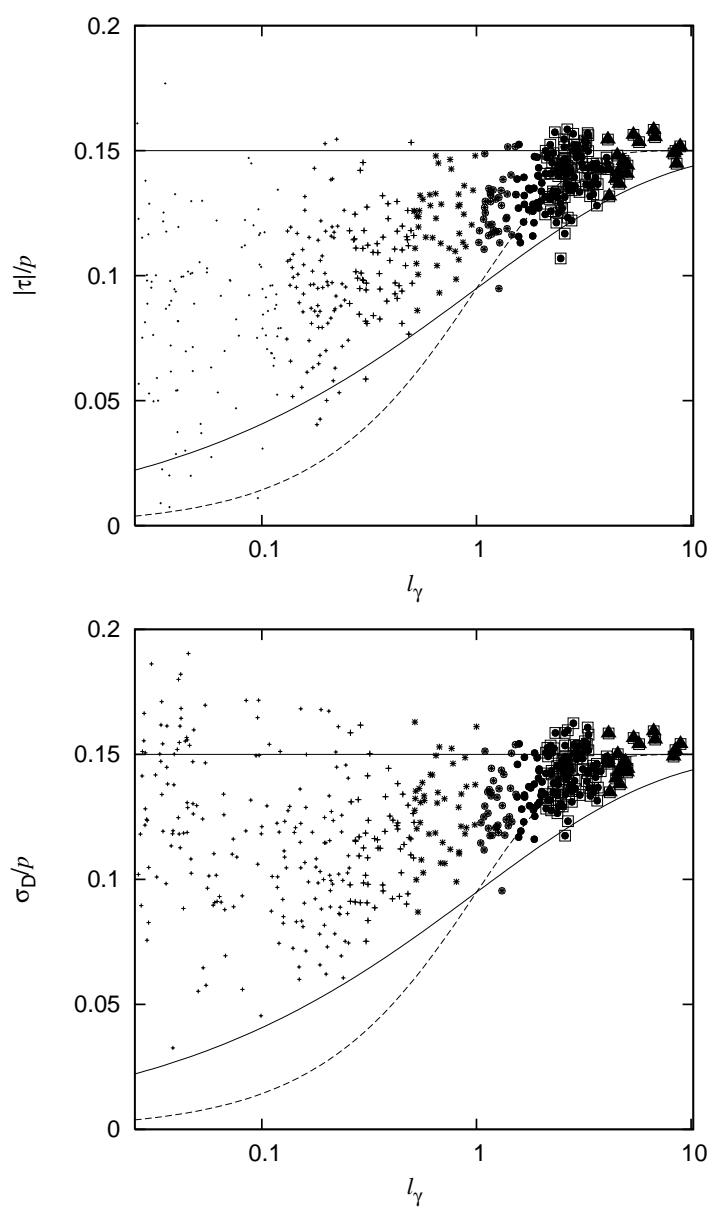

Figure 5: (Left) Shear stress intensity $|\tau| / p$, and (Right) anisotropy $s_{D}:=\sigma_{D} / p$, plotted against the shear path $l_{\gamma}$. The data for small pressures $p<100$ are disregarded here. The solid and the dashed lines correspond to Eq. (6), with $\alpha=0.5$ and $\alpha=1$, respectively. 
Differentiation with respect to $l_{\gamma}$ leads to a new incremental evolution equation:

$$
\frac{\partial s_{D}}{\partial l_{\gamma}}=\alpha l_{\gamma}^{\alpha-1}\left(\mu_{\text {macro }}-s_{D}\right),
$$

with $\alpha$ as a free parameter. In our case, the maximal deviatoric stress magnitude is approached by a stretched exponential with power $\alpha=0.5$.

\section{Conclusion}

Simulations of a split-bottom Couette ring shear cell show perfect qualitative and good quantitative agreement with experiments. Frictionless simulations are already in $80 \%$ percent agreement with the experiments - and the simulation with friction comes even close to $90 \%$, as was shown in Ref. (Luding, 2008b). This is remarkable, since besides the geometry of the shear cell no special attention was paid to the choice of material parameters, particle-size and particle size distribution.

From simulations (like from experiments) one observes that the shear-planes are tilted from the horizontal, as proposed in Ref. (Depken et al., 2006). The shear stress intensity is computed - under the assumption that the stress eigen-system is co-linear with the velocity gradient eigen-system - and compared to the objective stress anisotropy. The latter is always larger than the former, decreasing with strain rate to a ratio close to unity.

The stress anisotropy is limited by the macroscopic friction coefficient $\mu_{\text {macro }} \approx$ 0.15 . Shear planes with small shear path occur with all values, $\mu_{\text {macro }} \geq$ $s_{D} \geq s_{D}^{\min }$, where the stretching power $\alpha \approx 0.5$ is un-explained. Interestingly, the shear path $l_{\gamma}$ is not scaled by a relaxation length. The conclusion is that shear planes have to move relative to each other by a distance of the order of one particle diameter before the maximum anisotropy can be established.

\section{Acknowledgements}

We acknowledge the financial support of several funding institutions that supported this study, the Deutsche Forschungsgemeinschaft (DFG), and the Stichting voor Fundamenteel Onderzoek der Materie (FOM), financially supported by the Nederlandse Organisatie voor Wetenschappelijk Onderzoek (NWO). Furthermore, very helpful discussions with M. van Hecke and D. Wolf are acknowledged. 


\section{List of Symbols}

Radial, angular and vertical coordinates

Contact force

Contact stiffness

Contact deformation (overlap)

Contact friction coefficient

Macroscopic friction coefficient and friction angle

Radial coordinate

Outer wall, slit-, and inner wall-radius

Center and width of the shearband

Rotation rate and angular frequency of the outer wall

Angular velocity and strain rate

Nondimensional strain rate

Shear path

Eigenvector perpendicular to the shear plane

Tilt of $\hat{\gamma}$ from the horizontal

Static stress tensor components

Eigenvalues of the stress tensor

Eigenvectors of the stress tensor

Shear stress and deviator stress magnitude $r, \phi$, and $z$

$f$

$k$

$\delta$

$\mu$

$\mu_{\text {macro }}$ and $\psi$

$r$

$R_{o}, R_{s}$, and $R_{i}$ $R_{c}$ and $W$

$f_{o}$ and $\Omega_{o}$

$v_{\phi}$ and $\dot{\gamma}$

$I$

$l_{\gamma}$

$\hat{\gamma}$

$\theta$

$\sigma_{\alpha \beta}$

$\sigma_{\max }, \sigma_{0}$, and $\sigma_{\min }$

$\hat{\sigma}_{\max }, \hat{\sigma}_{0}$, and $\hat{\sigma}_{\text {min }}$

$|\tau|$ and $\sigma_{\mathrm{D}}$

\section{References}

Allen, M. P., and Tildesley, D. J. 1987. Computer Simulation of Liquids. Oxford: Oxford University Press.

Depken, M., van Saarloos, W., and van Hecke, M. 2006. Continuum approach to wide shear zones in quasistatic granular matter. Phys. Rev. E, 73, 031302.

Fenistein, D., and van Hecke, M. 2003. Kinematics - Wide shear zones in granular bulk flow. Nature, $\mathbf{4 2 5}$ (6955), 256.

Fenistein, D., van de Meent, J. W., and van Hecke, M. 2004. Universal and wide shear zones in granular bulk flow. Phys. Rev. Lett., 92, 094301. e-print cond-mat/0310409.

Herrmann, H. J., Hovi, J.-P., and Luding, S. (eds). 1998. Physics of dry granular media - NATO ASI Series E 350. Dordrecht: Kluwer Academic Publishers.

Kishino, Y. (ed). 2001. Powders \& Grains 2001. Rotterdam: Balkema. 
Lätzel, M., Luding, S., and Herrmann, H. J. 2000. Macroscopic material properties from quasi-static, microscopic simulations of a twodimensional shear-cell. Granular Matter, 2(3), 123-135. e-print condmat/0003180.

Lätzel, M., Luding, S., Herrmann, H. J., Howell, D. W., and Behringer, R. P. 2003. Comparing simulation and experiment of a 2D granular Couette shear device. Eur. Phys. J. E, 11(4), 325-333.

Luding, S. 1998. Collisions \& Contacts between two particles. Page 285 of: Herrmann, H. J., Hovi, J.-P., and Luding, S. (eds), Physics of dry granular media - NATO ASI Series E350. Dordrecht: Kluwer Academic Publishers.

Luding, S. 2004a. Micro-Macro Models for Anisotropic Granular Media. Pages 195-206 of: Vermeer, P. A., Ehlers, W., Herrmann, H. J., and Ramm, E. (eds), Modelling of Cohesive-Frictional Materials. Leiden, Netherlands: Balkema. (ISBN 041536023 4).

Luding, S. 2004b. Molecular Dynamics Simulations of Granular Materials. Pages 299-324 of: Hinrichsen, H., and Wolf, D. E. (eds), The Physics of Granular Media. Weinheim, Germany: Wiley VCH.

Luding, S. 2005a. Anisotropy in cohesive, frictional granular media. $J$. Phys.: Condens. Matter, 17, S2623-S2640.

Luding, S. 2005b. Shear flow modeling of cohesive and frictional fine powder. Powder Technology, 158, 45-50.

Luding, S. 2006. Particulate Solids Modeling with Discrete Element Methods. Pages 1-10 of: Massaci, P., Bonifazi, G., and Serranti, S. (eds), CHoPS-05 CD Proceedings. Tel Aviv: ORTRA.

Luding, S. 2008a. Cohesive frictional powders: Contact models for tension. Granular Matter, 10, 235-246.

Luding, S. 2008b. The effect of friction on wide shear bands. Particulate Science and Technology, 26(1), 33-42.

Luding, S. 2008c. Objective constitutive relations from DEM. Pages 173182 of: Grabe, J. (ed), Seehäfen für Containerschiffe zukünftiger Generationen. TUHH, Germany: GB.

Luding, S., Lätzel, M., and Herrmann, H. J. 2001. From discrete element simulations towards a continuum description of particulate solids. Pages 39-44 of: Levy, A., and Kalman, H. (eds), Handbook of Conveying and Handling of Particulate Solids. Amsterdam, The Netherlands: Elsevier. 
MiDi, GDR. 2004. On dense granular flows. Eur. Phys. J. E, 14, 341-365.

Vermeer, P. A., Diebels, S., Ehlers, W., Herrmann, H. J., Luding, S., and Ramm, E. (eds). 2001. Continuous and Discontinuous Modelling of Cohesive Frictional Materials. Berlin: Springer. Lecture Notes in Physics 568. 